\title{
Louis Dirn
}

Laurence Duboys Fresney

Catherine Bidou

Louis Chauvel

Yannick Lemel

\section{Chronique des tendances de la société française}

In: Revue de I'OFCE. N49, 1994. pp. 229-246.

\section{Résumé}

Cette chronique traite de trois phénomènes sociaux : $\bullet$ Le chemin parcouru en matière de politique urbaine a conduit à la création de banlieues « en difficulté ». Les pouvoirs publics ont, dans un premier temps, fait face au logement d'une population croissante en aidant les collectivités à construire de grands ensembles (aide à la pierre) ; par la suite, ils ont remplacé ces subventions par des aides à la personne (habitat pavillonnaire), multipliant ainsi les risques de ségrégation des populations selon leur revenu. $\mathrm{m}$ Le changement de comportement des électeurs. Relativement aux électeurs inscrits, les partis à vocation gouvernementale mobilisent de moins en moins, suite à la montée en puissance simultanée des abstentions et des partis " hors système »; cette situation s'explique non pas par un déclin de l'intérêt pour la politique ou un rejet du clivage droite/gauche, mais par une divergence croissante entre les aspirations des électeurs et les impératifs de la gestion gouvernementale. $m$ Enfin une conséquence de la diminution de la taille des familles. On constate que la réussite scolaire est plus fréquente parmi les familles de deux enfants. La convergence vers le modèle de la famille à deux enfants pourrait avoir un impact sensible sur l'élévation du niveau scolaire des nouvelles générations.

Citer ce document / Cite this document :

Dirn Louis, Duboys Fresney Laurence, Bidou Catherine, Chauvel Louis, Lemel Yannick. Chronique des tendances de la société française. In: Revue de l'OFCE. N49, 1994. pp. 229-246.

doi : $10.3406 /$ ofce.1994.1368

http://www.persee.fr/web/revues/home/prescript/article/ofce_0751-6614_1994_num_49_1_1368 


\title{
Chronique des tendances de la société française
}

\author{
Louis Dirn *
}

Cette chronique traite de trois phénomènes sociaux:

- Le chemin parcouru en matière de politique urbaine a conduit à la création de banlieues "en difficulté ". Les pouvoirs publics ont, dans un premier temps, fait face au logement d'une population croissante en aidant les collectivités à construire de grands ensembles (aide à la pierre); par la suite, ils ont remplacé ces subventions par des aides à la personne (habitat pavillonnaire), multipliant ainsi les risques de ségrégation des populations selon leur revenu.

- Le changement de comportement des électeurs. Relativement aux électeurs inscrits, les partis à vocation gouvernementale mobilisent de moins en moins, suite à la montée en puissance simultanée des abstentions et des partis "hors système"; cette situation s'explique non pas par un déclin de l'intérêt pour la politique ou un rejet du clivage droite/gauche, mais par une divergence croissante entre les aspirations des électeurs et les impératifs de la gestion gouvernementale.

- Enfin une conséquence de la diminution de la taille des familles. On constate que la réussite scolaire est plus fréquente parmi les familles de deux enfants. La convergence vers le modèle de la famille à deux enfants pourrait avoir un impact sensible sur l'élévation du niveau scolaire des nouvelles générations.

\section{Des politiques urbaines à contre-temps}

On parle toujours de banlieues "chaudes " ou "en difficulté " sans plus de précisions mais ce ne sont pas n'importe quelles périphéries urbaines qui s'embrasent périodiquement.

Les banlieues traditionnelles, même d'apparence rébarbative ou mal équipées, sont rarement celles qui font parler d'elles. Les plus explosives se trouvent surtout parmi les vastes programmes de financement du

- Le pseudonyme Louis Dirn désigne une équipe de sociologues qui se réunit à l'OFCE. Ont collaboré à cette chronique : Catherine Bidou, Louis Chauvel, Yannick Lemel. L. Duboys Fresney a assuré la coordination de l'ensemble. 
logement et l'organisation de l'habitat dans l'espace, mis en œuvre durant les "Trente glorieuses». Les quatre cents quartiers récemment repérés comme "à problèmes " par les pouvoirs publics et qui doivent être l'objet de mesures spécifiques, correspondent majoritairement à ce type d'espace. Pourquoi ces grandes cités construites dans les années soixante et soixante-dix posent-elles ces problèmes? La réponse du sens commun serait qu'elles regroupent une forte proportion de ménages de faible niveau économique, dont un grand nombre sont issus de l'immigration, et que le chômage y sévit plus gravement qu'ailleurs. Mais en fait, beaucoup de ces quartiers appartiennent à de grandes agglomérations relativement protégées de la crise économique (lle de France, région lyonnaise), et, par contre, dans des régions très atteintes par la récession (Bassin houillier du Nord-Pas-de-Calais, sidérurgie Lorraine), on ne retrouve pas les mêmes manifestations de violence de la part des jeunes.

\section{Du « zoning " au pavillon}

Dès la deuxième moitié du dix-neuvième siècle, les villes françaises ont vu s'étendre leurs faubourgs mais ce n'est qu'après la Seconde guerre mondiale que furent mișes en place des politiques systématiques d'aménagement de l'espace urbain. La crise du logement était alors apparue de façon aiguë en raison des destructions dues à la guerre et de l'exode rural massif qui accompagna le développement industriel. C'est à cette époque que l'on érigea les premiers grands ensembles dans les périphéries des grandes villes. II fallait construire vite, beaucoup et le plus économiquement possible. Ces impératifs ont toujours été avancés pour justifier un urbanisme de type " grands-ensembles ", caractérisé par des bâtiments de grande taille, très uniformes selon des plans-masse rompant totalement avec toute tradition urbaine antérieure.

Diverses mesures prises entre 1957 et $1967^{(1)}$ témoignent d'une prise de responsabilité de la part d'un État jusqu'alors très peu interventionniste en matière d'organisation de l'espace ${ }^{(2)}$. À travers elles, on a voulu créer des réserves foncières en gelant les prix des terrains dans des périmètres déterminés afin de pouvoir construire en fonction de l'évolution des besoins. De 1958 à 1975, le nombre annuel de constructions neuves passa de 291000 à 514000 logements pour des populations toujours plus nombreuses à quitter les campagnes, et celles chassées

(1) En 1957 furent votées les lois-cadres sur les SDAU (schéma directeur d'aménagement et d'urbanisme) les ZUP et ZAD (zones à urbaniser en priorité et zones d'aménagement différé) et $\mathrm{Zl}$ (zones industrielles), lois auxquelles en 1958 on ajouta des décrets regroupés sous l'intitulé « Urbanisme, HLM, crise du logement ". D'autres décrets et ordonnances d'application se succéderont pendant une dizaine d'années pour compléter ces mesures législatives. La loi d'orientation foncière de 1967 met quasiment fin aux procédures de ZUP jugées trop rigides et organise les ZAC (zones d'aménagement concerté).

(2) Elles s'inspiraient également pour partie de politiques foncières déjà mises en place dans la plupart des autres pays occidentaux. 
par les rénovations des centres-villes historiques ${ }^{(3)}$. Ces contraintes étaient aussi celles d'autres pays voisins, mais la France, restée plus tardivement rurale, eut à faire face à une urbanisation plus soudaine donc plus difficile à maîtriser. Cet argument ne suffit cependant pas à justitifier la médiocrité architecturale et l'incurie urbanistique qui présidèrent à l'édification des nouveaux ensembles d'habitat programmé.

Ceux-ci furent très marqués par l'influence de Le Corbusier sur les professionnels de cette époque, alors que lui-même ne construisit que très peu à l'intérieur de l'hexagone. II avait été un des plus fervents artisans de l'approche fonctionnaliste de la ville et de la théorie du "zoning " ${ }^{(4)}$. Celle-ci avait pour fondement l'inscription de chacune des fonctions urbaines dans un espace spécialisé. Abandonner la conception traditionnelle de bâtiments alignés le long de rues et construire des unités de grandes taille libérait le sol au profit d'espaces verts. Ces conceptions, innovantes sur le plan architectural, étaient très critiquables sur le plan urbanistique et furent très justement critiquées. Les réalisateurs des quartiers nouveaux firent du mauvais Le Corbusier, sans même en retenir les éléments les plus positifs comme la qualité des espaces intérieurs et l'introduction de services collectifs au sein des unités d'habitation.

A l'époque peu de voix s'élevèrent, même "à gauche ", pour dénoncer ces partis architecturaux et urbanistiques. La critique urbaine, pertinente au demeurant, portait exclusivement sur le cadre capitalistique de production. Les architectes qui osaient critiquer les caractéristiques formelles de ce type d'habitat étaient traités de rétrogrades, si ce n'est de réactionnaires ${ }^{(5)}$.

Pour éviter certains inconvénients des premiers grands ensembles du début des années cinquante (une trop grande homogénéité sociologique, et une pénurie d'équipements), il était prévu de "mixer" des types d'occupation, locatif et accession à la propriété, et des types de logements, afin d'éviter la formation de ghettos sociaux. Dans la majorité des cas, on n'atteint pas cette diversité car les investisseurs privés, censés construire pour les cadres, étaient rares dans ces zones.

Certaines ZUP (zones à urbaniser en priorité) parvinrent avec les années à s'intégrer au reste du tissu urbain et se transformer en quartiers

(3) Paris, Lyon, Marseille furent les villes les plus profondément remaniées dans les années soixante et soixante-dix, que ce soit à travers des rénovations ou des réhabilitations. Mais ces opérations ont eu en commun de contraindre les populations originaires à quitter les lieux. Ces tranferts ont été rendus possibles par des offres de logement social en banlieues. Cf. l'étude de $\mathrm{H}$. Coing sur le Treizième arrondissement de Paris, Rénovation urbaine et changement social, Les Editions ouvrières, 1967.

(4) Que l'on trouve théorisée dans plusieurs ouvrages de Le Corbusier, La Charte d'Athènes, issue du Congrès International d'Architecture Moderne (CIAM) de 1933, première édition Paris 1941, Editions Points Le Seuil, 1971 ; et également Quand les cathédrales étaient blanches, Editions Plon, 1937.

(5) Le ministre E. Claudius-Petit présentant les résultats d'un concours d'architecture à Strasbourg s'exprimait ainsi : "Une préoccupation semble avoir dominé toutes les propositions, conserver le plus d'espace libre possible et abandonner les formes surannées des villes qui chassent la nature en établissant un désert de pierres par ces blocs fermés, paresseusement alignés sur la rue bruyante que naguère encore nos pères construisaient. " Cité par R. H. Guerrand, 1989, Cent ans d'habitat social, Albin Michel. 
presque comme les autres. Parfois elles ont même pu constituer un habitat recherché. Dans certains ensembles d'habitat social, des catégories relativement aisées, qui avaient pu jouer sur les critères d'attribution et profiter de conditions de logement avantageuses, étaient même surreprésentées ${ }^{(6)}$. Dès la fin des années soixante (alors qu'on était en période de plein emploi), ces cités devinrent le théâtre de phénomènes de dégradation et de délinquance qui rendaient pénible la vie quotidienne des habitants et en faisaient fuir beaucoup dès la première occasion. Une décennie après leur démarrage, de nombreuses ZUP et grands ensembles étaient devenus des "problèmes sociaux" (cf. La Courneuve au nord de Paris et son grand ensemble de 4000 logements, Le Haut du Lièvre à Nancy, 400 mètres de façade, la ZUP $n^{\circ} 1$ à Marseille, 10000 logements, pour ne citer que les cas les plus connus). II fallut déjà envisager leur " réhabilitation ".

Dans le même temps (en partie en raison des "problèmes " engendrés par l'urbanisme de grands ensembles), les pouvoirs publics révisaient leurs points de vue. Le Livre Blanc des HLM, le Rapport Barre de 1976, dressaient les mêmes constats : les revenus vont croître, l'inflation va durer (ils tablaient sur 2,5\% annuels de croissance de pouvoir d'achat et $8 \%$ de hausse des prix), les mécanismes antérieurs de financement doivent être repensés, l'État se désengage. La volonté de désengagement de l'État conduisit à préférer aux aides à la pierre, les aides à la personne, donc à remplacer des subventions à l'investissement, données définitivement, par des versements aux personnes, à caractère temporaire, puisqu'ils diminuaient au fur et à mesure que les revenus de cellesci augmentaient. La loi de 1977 instaura l'aide personnalisée au logement (APL), banalisa les financements de l'accession à la propriété sociale (prêts PAP). Le logement social allait être moins directement pris en charge par des budgets publics; mais des aides appropriées permettraient aux ménages d'avoir accès à des logements de meilleure qualité, en secteur privé ou public.

Ce changement de politique contribua largement à un développement de l'accession à la propriété pavillonnaire. Celle-ci avait déjà eu les faveurs du ministère Chalandon (1969), elle connut une nouvelle impulsion à travers les systèmes de prêts avantageux que constituaient les PAP (pouvant être complétés par les APL). A cette époque les pouvoirs publics interprétaient le rejet du HLM et du grand ensemble comme celui de la promiscuité dans l'habitat collectif. La préférence du "Français" pour la maison individuelle était publiquement affirmée ${ }^{(7)}$. On vit fleurir sur le marché de la promotion immobilière de nombreuses entreprises offrant du pavillon "pré-construit industriel », produit particulièrement adapté à ce nouvel arsenal législatif et financier ${ }^{(8)}$. Les zones péri$n^{\circ} 49$.

(6) P. Clerc, 1967, Grands ensembles, banlieues nouvelles, Travaux et Documents INED

(7) P. Bourdieu, M. de Saint-Martin, 1990, * Le sens de la propriété, la genèse sociale des systèmes de préférences ", Actes de la recherche en sciences sociales, n० 81-82, mars.

(8) Quelques années plus tard la crise économique et la montée du chômage entraînèrent dans la précarité de nombreux ménages engagés trop légèrement dans l'accession. Mais dans ce type d'habitat les problèmes sociaux sont moins visibles car plus difficilement exprimables. Cf V. Monal, 1989, "Le prix du rêve", Informations sociales $n^{\circ} 2-3$. 
urbaines et en particulier les communes rurales appartenant à des ZPIU (zones de peuplement industriel et urbain) connurent dans ces années-là une expansion démographique exceptionnelle à travers l'éclosion débridée d'une multitude de lotissements pavillonnaires ${ }^{(9)}$.

\section{Vers la ségrégation sociale}

Cette faveur soudaine de l'accession à la propriété pavillonnaire entraîna chez les décideurs un désintérêt prolongé pour les quartiers de grands ensembles. Les catégories socio-économiques les plus aisées avaient dès les premières années commencé à les quitter. Ce processus de fuite sélective fut accéléré dans les années qui suivirent la promulgation des lois Barre. Le phénomène entraîna une accélération de la détérioration des conditions de vie.

Malgré les difficultés qui avaient marqué leurs débuts, la plupart des ZUP avaient vu éclore durant des années une vie associative relativement intense. Grâce aux associations, aux militantismes, aux luttes diverses nombreuses durant les années soixante-dix sur le terrain de l'habitat et du cadre de vie - furent construits, ici tel équipement, école, stade, là telle voie d'accès permettant une liaison plus commode avec le reste de la ville, fut repeint ailleurs un escalier ou refaite une façade, replanté un jardin, organisées des activités pour enfants etc. Mais au cours des années les leaders associatifs et les militants les plus actifs, qui appartenaient la plupart du temps aux catégories localement les plus favorisées, partaient habiter ailleurs, dans d'autres quartiers ${ }^{(10)}$.

Ces départs correspondaient à des itinéraires "normaux " de mobilité socio-professionnelle ascendante. De plus le souci d'une bonne scolarité pour leurs enfants, jugée improbable en demeurant sur place, poussa beaucoup de familles à déménager dès que leurs moyens financiers le leur permettaient; d'autant plus que l'institution scolaire était perçue de plus en plus par ces couches sociales (ouvriers qualifiés et classes moyennes), comme le vecteur exclusif de la promotion sociale.

Cette dynamique, une fois mise en place, fit "boule de neige ". Ne restèrent et ne restent désormais plus dans ces cités que des catégories de population ne pouvant plus prétendre, si rien n'est fait, à une quelconque ascencion sociale, ni même à une amélioration de leurs niveaux de vie: populations immigrées, attirées en masse dans les années soixante par les besoins en main-d'œuvre d'une industrie fordiste en plein essor ; populations d'origine métropolitaine rendues captives par leur précarité ou leurs très faibles revenus (type "petits fonctionnaires", employés

(9) J. Boudoul et J. P. Faur, 1982, “ Renaissance des communes rurales ou nouvelles formes d'urbanisation? "Economie et statistique, $n^{\circ} 149$, décembre.

(10) D. Mehl, 1980, "Les voies de la contestation urbaine". Annales de la recherche urbaine $n^{\circ}$ 6. M. Castells, E. Cherki, F. Godard, D. Mehl, 1978, Crise du logement et mouvements sociaux urbains, enquête sur la région parisienne. Mouton. 
municipaux...). Ces quartiers sont devenus progressivement ceux de la relégation, donc de l'exclusion, pour les ménages qui avaient en quelque sorte "raté leur examen de passage", et s'échouaient spatialement et symboliquement aux marges de ce vaste système que représentait la ville industrielle moderne. Les détériorations de l'habitat, une petite délinquance assez fréquente, apparaissent aux yeux de tous, et surtout des habitants eux-mêmes, comme les stigmates de cet échec.

Éléments de la logique résidentielle des années de politiques urbaines, les ZUP ont en effet joué à la fois un rôle de filtre et de propédeutique à la "vie moderne", du salariat et de la budgétisation des modes de vie quotidienne. On pourrait même dire que " tout s'est passé comme si " elles avaient été conçues dans ce double objectif, structurer les consommations des nouveaux venus, d'origine rurale ou étrangère, afin de les amener à vivre selon des modes de vie conformes, payant des loyers, des notes d'électricité et de chauffage, des traites de crédits pour l'ameublement et l'équipement correspondant à ces types de logements ${ }^{(11)}$. Ces habitudes prises, dans une conjoncture d'élévation générale des niveaux de vie, les ménages pouvaient passer à un nouvelle étape résidentielle ${ }^{(12)}$.

Mais la crise économique est venue gripper ces mécanismes. Au milieu des années quatre-vingt et pour la première fois depuis trente ans, les niveaux de vie de l'ensemble des catégories sociales n'étaient plus en croissance régulière mais en dents de scie ${ }^{(13)}$. Dans ces quartiers plus encore qu'ailleurs, le chômage a fait des ravages, entraînant une multiplication des difficultés des ménages. Pour beaucoup, la résidence en HLM se profila comme devant être définitive. Par ailleurs les mutations de l'appareil productif et le recul numérique des emplois ouvriers ont entraîné une décomposition de tout ce qui avait constitué la culture ouvrière. Cette déculturation s'est accompagnée, comme on le sait, d'un profond recul d'influence du parti communiste. Or les structures locales de cette organisation irriguaient et organisaient à bien des niveaux la vie quotidienne des habitants. Leur déliquescence s'est mêlée au recul du militantisme d'association pour laisser vide l'espace de la médiation sociale. Dans les années soixante, certains quartiers de grands ensembles avaient pu se constituer en territoires ouvriers, pratiqués et perçus comme tels ${ }^{(14)}$. Les mutations sociologiques profondes qu'allait connaître

(11) Ce thème de l'espace fordiste fut développé par A. Lipietz, 1977, Le Capital et son espace, Maspero.

(12) On a effectivement vu des cas où pour des grandes entreprises logeant son personnel, le passage en ZUP était obligatoire avant d'avoir le droit de revendiquer un pavillon. Cf. E. Campagnac, 1978, Transformations des modes de vie et habitat ouvrier, le developpement de la maison individuelle dans l'espace péri-urbain de Dunkerque, Plan Construction.

(13) Cf. les articles de G. Canceil, 1990, "Le revenu des ménages ", et de M. Moutardier,1990, "Consommation et niveaux de vie", Données sociales, INSEE. Cf également le rapport du CERC, 1989, Les Français et leurs revenus, le tournant des année quatre-vingt, La Découverte-La Documentation française.

(14) On pense ici par exemple à certains grands ensembles de La Seyne ou de SaintNazaire avant la crise des chantiers navals ou à certains grands ensembles qui ont progressivement succédé dans le Nord-Pas-de-Calais aux " maisons des mines", au fur et à mesure que les puits fermèrent et que des reconversions furent tentées. Cf $O$. Swartz, 1990, Le monde privé des ouvriers, hommes et femmes du Nord, Paris, PUF. 
l'ensemble de la structure sociale à partir des années quatre-vingt empêcheront que la plupart de ces espaces, surtout dans les plus grandes agglomérations, soient vécus positivement comme territoires identitaires.

L'heure était au triomphe de l'idéologie libérale et l'État-Providence mis en question. Aux élections municipales de 1983 beaucoup de villes, passées à gauche en 1977, firent un nouveau virage à droite. Au niveau communal de nombreux services ont été privatisés. Assistantes sociales et éducateurs restèrent en nombre insuffisant alors que les problèmes sociaux s'aggravaient.

Comportements et attitudes racistes, toujours latents, ont eu tendance à s'exprimer plus ouvertement du fait de l'isolement en face à face entre immigrés et "petits blancs". Ces derniers se sentaient plus que jamais captifs, sans perspective de mobilité vers un quartier plus gratifiant et plus conforme à leurs aspirations et modes de vie. L'“ immigré " est alors stigmatisé et devient un élément de fixation sur lequel se projettent rancœur et sentiment d'impuissance. D'autant plus que dans les cités, il vit souvent mieux que le "petit blanc". Les familles des "immigrés" sont plus nombreuses et plus solidaires, les salaires non pas plus élevés mais souvent pluriels dans un même foyer: les impayés de loyers et donc les risques d'expulsion sont plus rarement le fait de familles immigrées que de familles "françaises" précaires. Pourtant si les tensions raciales existent, les problèmes sociaux des cités ne sauraient cependant être réduits à elles. II y a des ZUP où il $n^{\prime}$ y a presque pas d'immigrés (certaines villes de Bretagne) et où le mécanisme de l'exclusion joue malgré tout. Ce qui n'empêche pas la nécessité d'une réflexion spécifique sur les problèmes qui concernent les "immigrés", les "communautés " (15).

Ce retour sur le passé des politiques de l'habitat en France depuis trente cinq ans fait ressortir le décalage entre la prise de conscience des problèmes, leur traduction en solutions techniques et l'application de celles-ci dans des contextes très différents. Dans un premier temps, les grands quartiers d'habitat programmé ont rempli leur fonction, ont eu leur cohérence tant qu'ils permettaient à divers types de ménages de parcourir diverses étapes résidentielles. Les arrivées à la ville ou les démarrages dans la vie se faisaient dans un premier temps en HLM, puis venaient le pavillon ou la résidence centre-ville quelques années plus tard. Mais ce système résidentiel correspondait au modèle économique dominant des années soixante et soixante-dix. Aujourd'hui ce modèle ne fonctionne plus et la hausse régulière des niveaux de vie n'est plus à l'ordre du jour. Cet habitat, sans la mobilité résidentielle qu'il impliquait, apparaît alors comme un piège. Le dispositif mis en place à la fin des années cinquante et durant les années soixante était efficace et rigide, trop rigide. Adapté à une période d'urgence, il a mal vieilli. Par les mesures législatives prises à la fin des années soixante-dix, le désengagement de l'État devait introduire de la souplesse dans le fonctionnement du système. Or cette

(15) Cf. l'article de O. Roy, 1991, "Ethnicité, bandes et communautarisme ", dans la Revue Esprit, fév., où l'auteur s'insurge contre l'usage immodéré des notions d'ethnies et de communautés dans les débats concernant ces quartiers. 
souplesse est arrivée à contre-temps, dans un contexte économique déphasé et n'a pas eu les effets escomptés, c'est-à-dire permettre aux ménages de bien se loger dans des types de logement non imposés, cassant les logiques antérieures. Cette réorientation s'est traduite entre autres dans l'extension pavillonnaire périphérique et le désinvestissement de l'habitat social.

C. B.

\section{La double dérive de l'électeur}

\section{La dérive des partis}

L'érosion progressive, à des élections comparables, des suffrages exprimés au profit des abstentions, des votes blancs et nuls, et le morcellement de l'électorat de plus en plus réticent à "voter utile " (c'est-à-dire pour des partis en mesure de conquérir la majorité) furent les enseignement des législatives de 1993. Ces élections ont prolongé la série d'alternances successives intervenant depuis les législatives de 1981, et furent marquées par la mobilité des électorats ${ }^{(16)}$ : il s'agirait d'une modification de fond du comportement des électeurs, moins attachés aux partis dont ils sont la clientèle politique, moins engagés, plus dépolitisés, moins disposés à offrir un blanc-seing, et plus soucieux de juger au coup par coup ${ }^{(17)}$. A la lumière des dernières élections législatives du printemps 1993, nous sommes en mesure de mieux situer les tendances à l'œuvre.

Ces élections signalent, au-delà du renforcement des oscillations entre électeurs de gauche et de droite, la défection tendancielle de l'électorat disposé à voter pour les " partis à vocation gouvernementale ": le PS, d'une part, et la droite parlementaire, de l'autre, qui joutent depuis 1958 pour la conquête du pouvoir ${ }^{(18)}$.

Pour la première fois depuis 1958 à un premier tour d'élections législatives, les partis à vocation gouvernementale disposent ensemble des suffrages de moins de la majorité des électeurs inscrits : la gauche et

(16) Voir Louis Dirn, 1991, "Chronique des tendances de la société française ", Observation et diagnostics économiques, Revue de l'OFCE, $n^{\circ} 35$.

(17) Voir Daniel Boy et Nonna Mayer, 1988, "L'électeur est-il stratège ? " in L'électeur français en question, Presses de la FNSP, Paris, pour un point sur la question de l'" électeur stratège $n$.

(18) Dans cette perspective de longue durée, le Parti Communiste occupe une position très particulière : les accords de désistements automatiques scellés lors de l'Union de la Gauche ont fait du PC un parti à vocation gouvernementale - au point de voir 4 ministres représenter le PC dans le gouvernement Mauroy de 1981-1984 —; depuis 1984, l'érosion du $\mathrm{PC}$, et sa stratégie propre vis-à-vis du PS modifient les données. 
la droite centristes représentaient $62 \%$ des inscrits en 1968 (et $78 \%$ si l'on ajoute le PC à ce décompte des votes "utiles"), ils ne concernent plus que $42 \%$ de l'électorat potentiel (et $48 \%$ en comptant le PC), au terme d'un déclin continu. Les partis “d'alternative " ${ }^{(19)}$ se développent au même rythme saccadè que les abstentions, les votes blancs et nuls, montrant ainsi les risques tendanciels de marginalisation des structures du politique telles qu'elles sont conçues depuis 1958 : entre droite parlementaire et sociaux-démocrates.

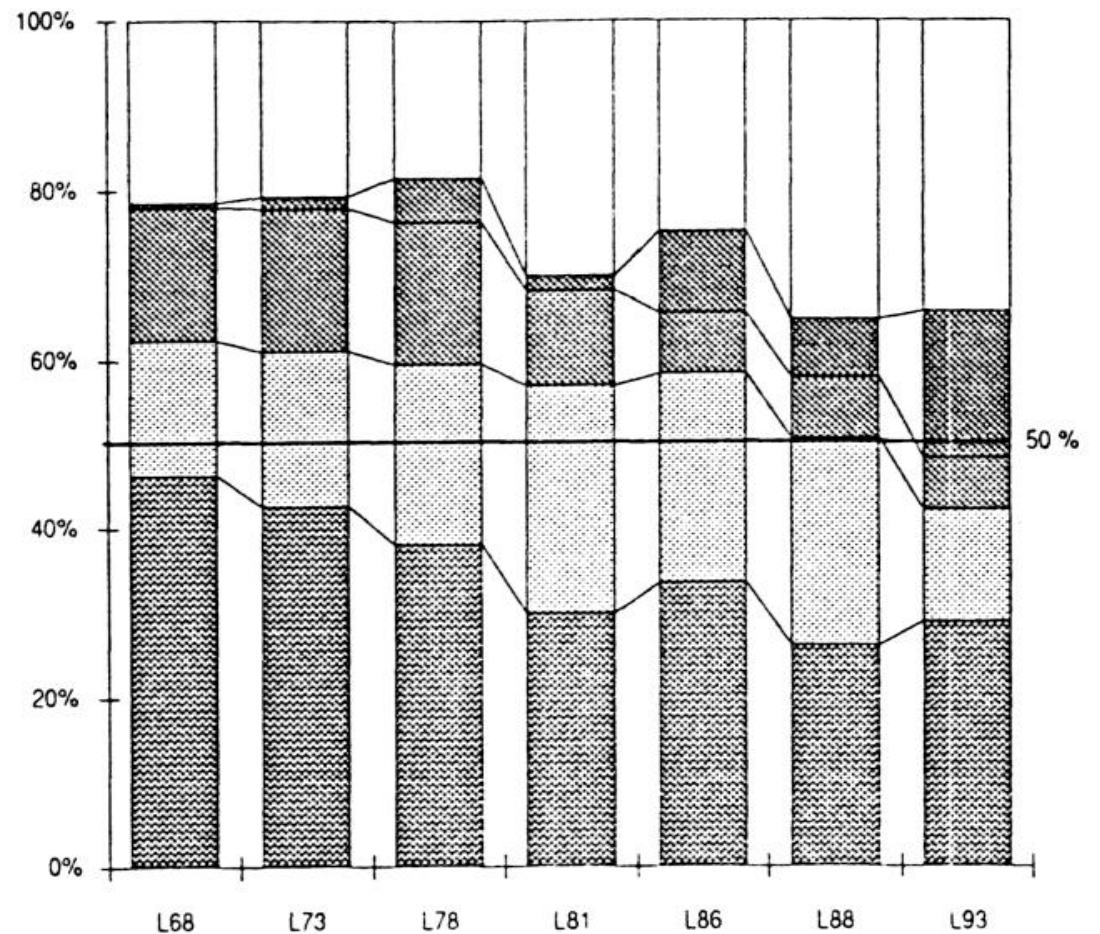

Source: C. Ysmal, 1986, Le comportement électoral des Français, Paris, La Découverte ; pour l'abstention et la non participation : A. Lancelot, 1983, Les élections sous la ve Republique, PUF, Paris, et F Subileau et M. F. Toinet, 1993, Les chemins de l'abstention, Paris, La Découverte; prolongation 1988 et 1993 : Etudes politiques du Figaro et Etat de l'opinion 1994, Le seuil, Paris.

\section{1. Évolution des parts respectives des types de votes parmi les inscrits aux premiers tours des législatives}

Plus finement, à côté du chassé-croisé de la gauche et de la droite parlementaires, nous constatons l'oscillation entre les abstentions et les partis "hors-système ", qui ne prétendent pas à la conquête du pouvoir : 1978, 1986 et 1993 marquent (relativement à la tendance générale) un recul des abstentions, et la croissance des électorats « hors-système "; le cumul du PC, des écologistes et de l'extrême gauche en 1978, la montée en puissance du Front national aux législatives de 1986, et le retour des écologistes à celles de 1993 - même si leur résultat fut un échec, au regard des pronostics électoraux, leur ré-émergence est néanmoins un événement politique remarquable - montrent bien, sur le

(19) D'autres qualifieraient ces mêmes partis de " hors-système "; outre le PC, qui est semble-t-il passé d'un statut de parti de gouvernement à la condition de parti de vote protestataire, nous qualifions là les partis d'étiquette extrémiste, de rupture ou de dépassement des clivages traditionnels, tels le PSU, les écologistes, le Front National. 
graphique, que la baisse enregistrée des abstentions, à ces échéances électorales, n'a profité qu'aux partis “ hors-système ". Par ailleurs, les premiers tours de législatives de 1981 et 1988 marquent une forte progression des abstentions et des suffrages blancs et nuls partiellement compensés par la baisse des partis d'alternative. Au total, quoi qu'il arrive, abstentions et vote pour les partis "hors-système " prennent des voix aux "partis à vocation gouvernementale ".

La tendance longue est donc un déclin de la mobilisation en faveur des partis classiques, déclin propice à l'alternance politique : en 1968 et 1973, la droite parlementaire obtenait plus de $40 \%$ des suffrages des inscrits dès le premier tour, configuration interdisant toute alternance; avec environ $30 \%$, comme depuis 1981, tout dépend de la mobilité des électeurs, des reports et des alliances. En 1993, au premier tour des législatives, la droite parlementaire (malgré un nombre de sièges exceptionnel, et une amélioration de son score au regard de 1988), fait un score inférieur à celui de sa défaite de 1981 , relativement aux inscrits $30,5 \%$ des inscrits en 1981, 29 en 1993. Tour à tour, les majorités se perdent, faute de rencontrer les suffrages de leur clientèle politique : audelà de la pratique de l'abstention, une multitude de partis sont susceptibles d'offrir à chacun la possibilité de moduler l'expression de sa sensibilité.

\section{La dérive du clivage droite et gauche}

Une telle évolution des électorats est attribuée, souvent, à un dépassement de la distinction entre gauche et droite, les électeurs se défiant de plus en plus de ces étiquettes et cherchant " autre chose "; l'examen attentif des données conduirait plutôt à constater un écart grandissant entre aspirations des électeurs et pratiques politiques des appareils partisans.

Les Français, en effet, éprouvent de plus en plus de difficultés à comprendre les comportements et les décisions de leurs représentants au travers d'une grille de lecture droite-gauche : les opinions favorables à l'idée que "les notions de droite et de gauche sont dépassées : ce n'est plus comme cela que l'on peut juger les prises de position des partis et des hommes politiques " passent en effet de $33 \%$ en mars 1981 à $55 \%$ en avril $1991^{(20)}$.

S'il apparaît donc aux électeurs que les partis sont moins repérables en termes de droite et de gauche, le dépassement du clivage droitegauche n'apparaît pas aussi unilatéral dans les consciences : Guy Michelat montre que, face aux échelles politiques proposées lors des sondages

(20) Voir, R. Cayrol, 1992, “La droite, la gauche, et les références idéologiques ", in L'état de l'opinion, Paris, Sofres - Editions du Seuil. Un dossier récent fait le tour de la question en France et à l'étranger : Etienne Schweisguth, 1994, Droite - Gauche : un clivage dépassé ? La Documentation française, Paris. 
d'opinion, les refus de réponse ont décliné entre les années soixante et la fin des années quatre-vingt ${ }^{(21)}$; un tel étiquetage politique reste ancré dans la culture politique des Français, au moins jusqu'à la fin des années quatre-vingt.

L'analyse rétrospective de la période 1977-1993 des sondages d'Agoramétrie (figure 2) fait apparaître les conséquences des revirements politiques de la période récente: l'indifférence à l'échelle droite-gauche a décru continuellement entre 1977 (38\% d'indifférents) et 1988 (22\%), au profit, généralement, de l'engagement à droite (de $12 \%$ en 1977, cet engagement est monté à près de $25 \%$ dans la période récente). La crise du début des années quatre-vingt-dix a mis à mal la gauche au profit des " indifférents", mais il se peut que ce soit là plus un effet de conjoncture que de modification des structures: la période 1984-1985 (qui correspond à la fin de l'état de grâce, à la mobilisation remarquable autour de la question de l'école privée, au recul momentané du pouvoir d'achat des ménages, mais aussi à la diffusion d'un pessimisme important, qui fut propice au développement de l'extrême-droite) montre, pour l'engagement à gauche, un recul momentané aussi important que dans la période récente. La superposition des effets de crise (le corps social supportant mal, en France, une croissance nulle du pouvoir d'achat et une croissance des problèmes d'emploi) et d'erreurs politiques (les "affaires ") ont pour effet, en 1984-1985 comme en 1990-1992, de sanctionner lourdement la majorité en place.
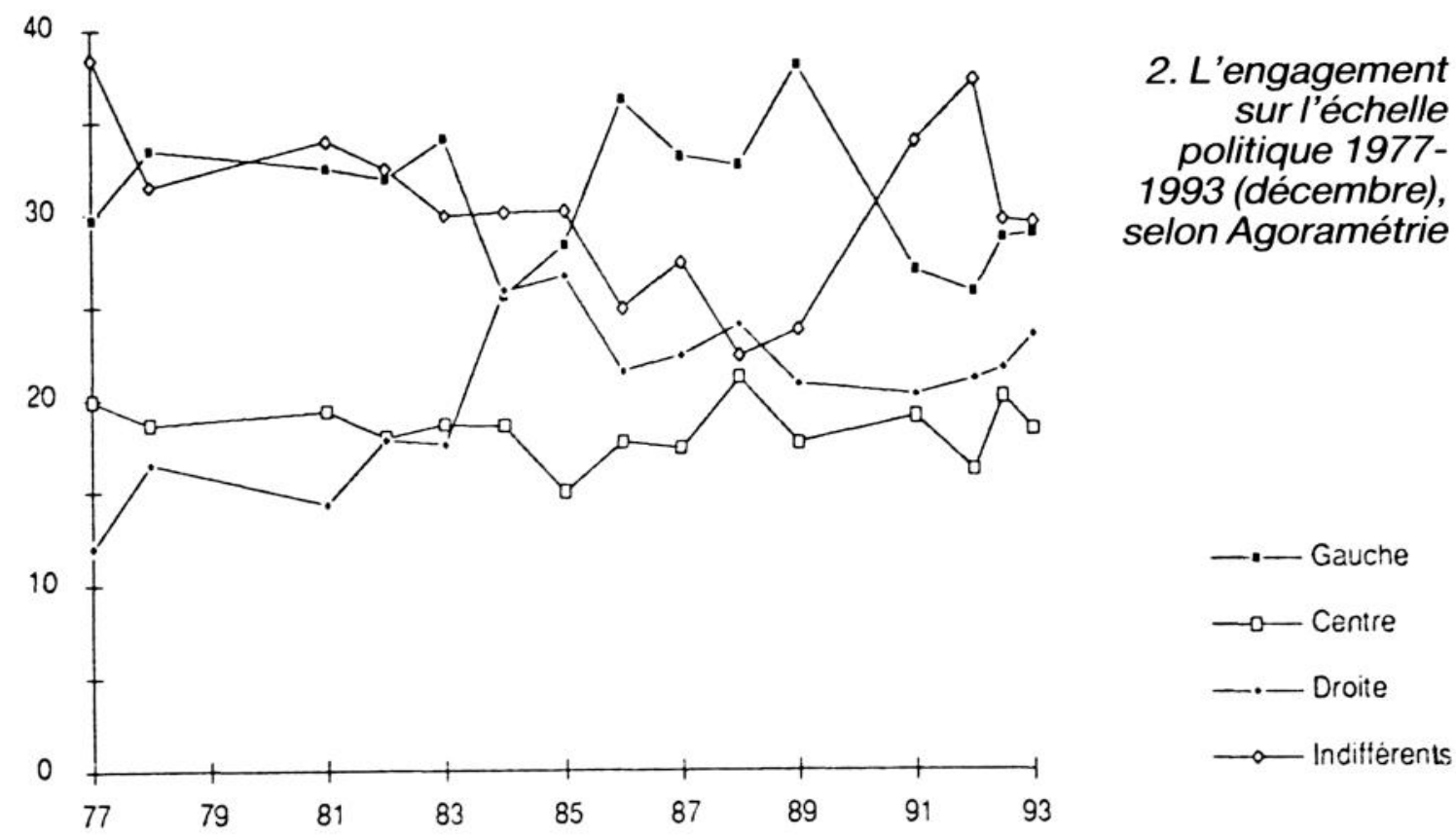

Source: Banque de données Agoramétrie ; analyse rétrospective.

(21) G. Michelat, 1990, “ A la recherche de la gauche et de la droite ", dans L'électeur français en questions, Presses de la FNSP. Guy Michelat fait une étude rétrospective de 1964 à 1988 sur l'échelle de la Sofres en sept modalités : extrême gauche / gauche / centre gauche / centre / centre droite / droite / extrême droite. 
Une typologie des années 1977 à 1993 selon l'engagement sur l'échelle droite-gauche des catégories socioprofessionnelles sur (et hors de) l'échelle politique (22) montre l'existence de trois périodes homogènes :

- 1977 à 1983 : la gauche est majoritaire, les enseignants, les étudiants, les employés et ouvriers sont fortement engagés à gauche, et les indépendants sont à droite, mais réticents à s'engager sur l'échelle politique ;

- 1984-1985 : la gauche est «lâchée » par les employés-ouvriers, et la droite remobilise les indépendants, cadres supérieurs et retraités ;

- 1986 à 1989, années particulières d'apaisement et de moindre désillusion devant le politique : les refus d'engagement sur l'échelle sont moindres, la gauche redevient crédible auprès des électorats populaires;

- 1991-1993 : retour à une situation proche de 1984-1985, avec une gauche décrédibilisée dans l'électorat populaire, qui s'engage moins sur l'échelle politique et se situe plus souvent au centre ou à droite.

Au-delà du constat que ces évolutions observées entre 1977 et 1993 coïncident assez bien avec la conjoncture économique (la croissance étant généralement favorable à la gauche, la récession lui étant défavorable), les mouvements des catégories socioprofessionnelles révèlent la modification du débat entre le début des années quatre-vingt et celui des années quatre-vingt-dix (figure 3) : l'ensemble des catégories socioprofessionnelles qui formaient le soutien "naturel" de la gauche quittent l'échelle politique, ou se situent moins à gauche, pour ceux qui acceptent encore de s'y engager. En définitive, les enseignants sont les seuls, maintenant, à se situer massivement à gauche, formant ainsi une gauche intellectuelle isolée, abandonnée par l'ensemble des ouvriers, employés, étudiants. Les professions intermédiaires sont maintenant aussi souvent à gauche que les ouvriers, alors que, naguère, elles étaient relativement plus à droite ${ }^{(23)}$. De l'autre côté de l'échiquier politique, les catégories traditionnellement à droite s'engagent encore plus à droite et acceptent mieux de s'engager sur l'échelle politique.

(22) Ce constat provient de l'exploitation de la banque de données Agoramétrie, possédant une échelle politique en cinq modalités : extrême gauche / gauche / centre / droite / extrême droite, et refus d'engagement, indifférence. L'inconvénient d'une telle échelle est de donner une moindre place aux partisans modérés du centre droit et du centre gauche : cette échelle en cinq modalités produit donc une majoration des gens se situant indifféremment au centre, ou refusant de s'y inscrire. Cet inconvénient est tout relatif puisque nous nous intéressons aux évolutions comparées et non aux données brutes.

Quant aux catégories socioprofessionnelles, elles sont codées en deux chiffres dans la base de données Agoramétrie ; il nous a donc été possible de recoller les catégories socioprofessionnelles de I'INSEE de type CSP d'avant 1982 aux catégories PCS de façon homogène. Nous considérons ainsi les agriculteurs, les commerçants-artisants-industriels, les cadres supérieurs, les enseignants (professeurs et instituteurs), les étudiants, les professions intermédiaires (sans les instituteurs, mais avec les contremaîtres), les employés (définition PCS), les ouvriers, les retraités, les inactifs d'âge actif non-étudiants.

(23) Ce rapprochement entre ouvriers, employés et catégories intermédiaires du salariat, et l'homogénéisation partielle qui en découle, sont l'un des arguments majeurs en faveur de la baisse du déterminisme du choix politique par la catégorie sociale (voir R. Inglehart, 1987, "Values in Industrial Societies", American Political Science Review, Washington). Une telle affirmation n'est pas encore vraiment établie, puisqu'une autre interprétation conduit à dire que les classes moyennes salariées s'éloignent des indépendants et des retraités en général, pour former un bloc salarié opposé aux propriétaires; cette interprétation est alors radicalement différente de celle que l'on souhaitait établir: la fin de la lutte des classes.. 
Au bout du compte, à part les enseignants, le bord gauche de l'échiquier politique s'est vidé. La distinction entre les catégories salariées moyennes et les strates populaires au regard de l'appartenance à la gauche est annulée. Enfin, si l'on excepte les étudiants, qui trouvent dans l'écologie l'alternative politique qui leur convient, et les agriculteurs dont le regain d'engagement est vraisemblablement lié aux problèmes économiques récents, qui ont braqué sur eux les feux de l'actualité l'engagement sur l'échelle politique reproduit la hiérarchie socioprofessionnelle, entre les cadres supérieurs et professions libérales dont l'engagement sur l'échelle politique reste important en toute condition, et les strates populaires, incapables de trouver leur place dans le jeu politique, tout comme les inactifs d'âge actif (ou, très majoritairement, les inactives, c'est-à-dire les femmes au foyer).

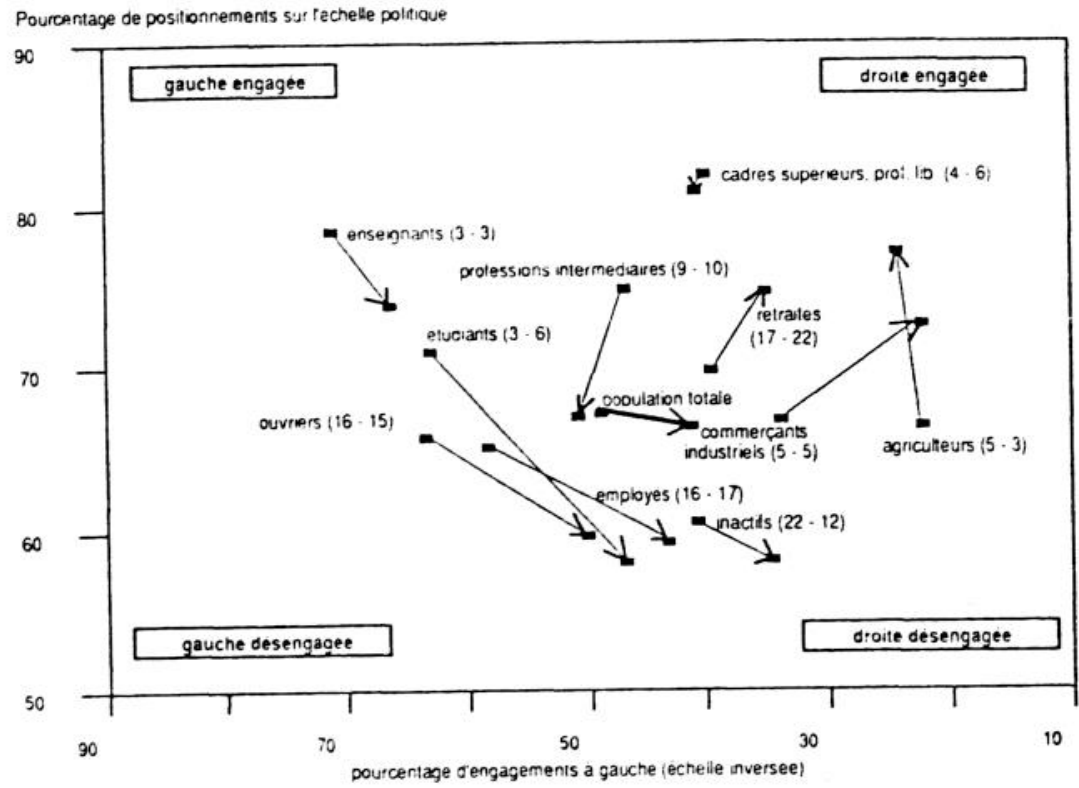

\section{L'engagement surl'échelle politique 1977- $1983(n=6288)$ à $1991-1993(n=$ 5015)}

Source : Banque de données Agoramétrie ; analyse rétrospective.

Note: en abscisse: pourcentage d'autoposition à gauche sur l'échelle parmi les individus a situe à gauche sur le individus acceptant de s'y placer (plus une categorie se situe à gauch a chez les graphique, plus sont fréquentes les autopositions à gauche : $70 \%$ chez les enseignants); en ordonnee: I acceptation de elle accepte de s'engager sur l'échelle : situee dans la partie haute du graphique, plus se positionner sur l'échelle). Entre $80 \%$ des cadres superieurs acceptent de parenthèses: estimation du pourcentage de la population concernè aux duactivité périodes ; la baisse drastique des " inactifs " est lié à l'augmentation du taux d'activité des femmes et à l'abaissement de l'âge de la retraite (à 60 ans dans la nouvelle
nomenclature), conduisant à reclasser les inactif(ve)s de plus de 60 ans en " retraités ".

En résumé, les évolutions globales de l'engagement dans ou hors de l'échelle politique dans la période contemporaine sont relativement faibles au regard de la fin des années soixante-dix: l'indifférence à l'échelle politique culminait à $38 \%$ en 1977 , et touchait $37 \%$ des citoyens en 1992 ; le dépassement du clivage droite-gauche est donc tout à fait relatif. En revanche, les représentations politiques de chaque catégorie professionnelle se sont considérablement modifiées entre les deux périodes, dans le sens d'un rééquilibrage entre sentiment d'appartenance à droite et à gauche. 
Deux explications peuvent être confrontées devant la situation présente : effet transitoire d'une crise de croissance et d'une inquiétude grandissante devant le chômage, décrédibilisant la gauche, ou modification durable des électorats "naturels" de la gauche et de la droite. La seconde interprétation semble meilleure puisque que le schéma " gauche populaire contre droite indépendante " cède le pas à une distinction entre l'engagement politique fort des catégories aisées de la population, contre une indifférence croissante des électorats dont l'insertion économique et sociale est difficile.

Cette évolution est corroborée par la modification des valeurs des Français: au vu de nombreuses analyses, il apparaît aussi que la nature et la signification, pour le citoyen, des termes de droite et de gauche se sont modifiées en quinze ans. II semble d'une part que la composante "anticapitaliste" de la gauche a perdu un terrain considérable au début de la décennie quatre-vingt ${ }^{(24)}$ (d'où le fort déclin de la gauche populaire), mais qu'en revanche la gauche que l'on qualifie d'" humaniste" (la gauche anti-autoritaire, vigoureusement opposée à la peine de mort, à la xénophobie, favorable aux aides en faveur des pays du Tiers Monde) a connu un renforcement de sa cohérence idéologique; or, cette gauche-là n'est guère suivie par sa base.

Néanmoins, l'étude suivie sur l'ensemble des années quatre-vingt incite à interpréter ces mouvements comme des oscillations pendulaires de défections et de réengagements, se retournant à chaque alternance politique, au profit de l'opposition gouvernementale et à la défaveur de la majorité, mouvement pendulaire d'autant plus puissant que la conjoncture économique est défavorable; si la droite parlementaire a tenu le pouvoir au long des années soixante-dix, la raison en est, vraisemblablement, l'avance dont elle disposait, suite à l'héritage d'un confortable capitalconfiance acquis lors de la seconde moitié des "trente glorieuses", et de l'image emblématique laissée par l'ère gaullienne.

La figure 1 montre bien que la structure n'est plus la même, et aucun bloc ne dispose d'une avance considérable sur l'autre, surtout une fois tenu compte de la mobilité des électorats flottants. Les suites d'alternances et de mouvements pendulaires, qui touchent particulièrement les catégories socioprofessionnelles faiblement diplômées, montrent surtout que la participation et le repli hors du politique relèvent avant tout du discrédit et de l'usure au pouvoir des équipes qui se succèdent.

II apparaît surtout qu'entre les idéologies et les aspirations des électorats qui portent une équipe au gouvernement, et les contraintes économiques - que cette équipe devra gérer, un écart croissant se forme. La baisse tendancielle du nombre des électeurs sur lesquels se font les nouvelles majorités gouvernementales provient moins de ce que le clivage entre gauche et droite décline - dans l'esprit des électeurs -, mais de ce que ces majorités doivent gouverner et gérer en dépit des choix de leurs électorats, qui font défection à mesure de l'" usure au pouvoir " de la majorité en place.

(24) Voir, notamment H. Riffault (dir.), 1994, Les valeurs des Français, PUF, Paris. 
Avec le temps, cette difficulté devient d'autant plus cruciale que, à l'évidence, les partis à vocation gouvernementale s'usent plus vite au gouvernement qu'ils ne reconstituent leur force dans l'opposition. Dans ce jeu, l'électeur apparaît moins stratège que sans repère.

L. C.

\section{L'élévation du niveau scolaire : effet imprévu de la baisse de la fécondité ?}

Le modèle de "famille à deux enfants" s'est généralisé dans les années d'après-guerre.

Tout donne à penser que cette tendance, que nous présentions dans La société française en tendances ${ }^{(25)}$, va se poursuivre et que les familles nombreuses comme les familles d'enfant unique seront de plus en plus rares. Aujourd'hui, la propension à avoir un enfant supplémentaire après en avoir eu au moins deux tombe de $60 \%$ pour les femmes nées dans les années trente à $50 \%$ pour celles nées dans les années quarante. S'il y a moins de femmes à ne pas avoir d'enfant, la baisse de la fécondité résulte de la raréfaction des familles nombreuses ${ }^{(26)}$. Mais les conséquences du phénomène ne sont pas encore complètement apparues car les évolutions démographiques ont une forte inertie.

Actuellement la majorité des enfants sont, en fait, issus de familles "nombreuses" (trois enfants ou plus). Si, aujourd'hui, du point de vue des parents, le modèle à deux enfants est sans doute la référence, du point de vue des enfants, la référence est la famille nombreuse. En effet, et en quelque sorte par construction même, la descendance des parents engendrant plusieurs enfants pèse plus dans la cohorte des enfants que la descendance des parents n'en engendrant qu'un petit nombre. Avoir été élevé dans une famille nombreuse était donc encore la règle pour une génération d'enfants nés autour des années soixante-dix. II n'en sera sans doute plus de même à terme.

\section{La taille de la fratrie n'est pas sans effet sur la réussite scolaire...}

Dans les années soixante-dix, Alain Girard ${ }^{(27)}$ écrivait en rendant compte de ses travaux sur la réussite scolaire: "II apparaît en toute

(25) Louis Dirn, 1990, La société française en tendances, Paris, PUF, tendance 3.2, p. 97.
(26) Desplanques G., 1994, “Taille des familles et milieu social ", Insee-Première, $n^{\circ} 296$, tévrier.

(27) A. Girard 1971, La réussite sociale, Paris, PUF. 
certitude que les parents des personnalités contemporaines, aussi bien que des élèves des grandes écoles, ont eu nettement moins d'enfants que l'ensemble des couples constitués à la même époque ». Toutefois, ajoutait-il immédiatement : "Rien ne permet de conclure pour autant que la fécondité limitée de leur famille a été l'un des éléments déterminants de leur réussite...". Ces précautions sur la réalité d'un effet - l'importance de la fratrie nuit à la réussite scolaire - s'expliquent compte tenu des données dont disposait Alain Girard et du traitement qu'il avait pu en faire.

Peut-être s'expliquent-elles aussi par l'incrédulité que pouvait rencontrer la présentation d'effets de cette nature chez des lecteurs qui vraisemblablement avaient eux-mêmes été élevés dans des familles nombreuses.

Edith Blake examine dans son ouvrage Family Size and Achievement les résultats des différentes enquêtes réalisées aux Etats-Unis permettant de relier les caractéristiques sociales du milieu d'origine, la taille de la fratrie et différents indicateurs de réussite scolaire. Globalement la taille de la fratrie d'origine lui paraît l'un des facteurs explicatifs de la réussite scolaire, le plus important parmi ceux auxquels on peut penser, une fois contrôlé le niveau d'éducation des parents ${ }^{(28)}$.

Le panel de l'Education nationale, sans permettre des analyses aussi détaillées que celles menées par Edith Blake, car les informations disponibles sur les élèves et leurs ascendants sont moins nombreuses, fournit néanmoins pour la France d'aujourd'hui des résultats dont ne disposait pas Alain Girard.

Sur cent élèves entrés en sixième (à peu près donc toute la classe d'âge), $46 \%$ entreront en classe de seconde de l'enseignement général et $39 \%$ obtiendront le baccalauréat, résultat du suivi d'une cohorte d'élèves présents au cours préparatoire en 1980 et suivis tout au long de leur scolarité secondaire ${ }^{(29)}$. Les taux d'accès en seconde des membres de cette cohorte varient suivant la taille de la fratrie. Ils sont de $56 \%$ pour les enfants uniques, $57 \%$ pour les enfants d'une fratrie de 2 enfants, $47 \%$ pour ceux d'une famille de 3 et de $30 \%$ seulement si le nombre des enfants atteint ou dépasse les 4 . Les taux correspondants pour l'accès au baccalauréat sont de $47 \%, 47 \%, 39 \%$ et $23 \%$. Les différences sont donc très nettes. Bien entendu, la taille de la famille étant liée au milieu social d'origine qui conditionne lui-même très fortement la réussite, les différences peuvent résulter d'un " effet de struc-

(28) E. Blake, 1989, Family Size and Achievement, Berkeley, University of California Press. - Even after controlling for major parental background characteristics, we have found differences in total educational attainment - approximately two years of schooling - between small and large families that rival the simple difference by race and age ", indique-t-elle.

(29) Voir, pour plus de détails. Paul Esquieu, 1990, "Qui devient bachelier? " Education et formation, $n^{\circ} 23$, avril-juin. 
ture " - c'était le propos d'Alain Girard - et le tableau 1 permet de voir ce qu'il en est pour l'entrée en seconde.

1. Le taux d'entrée en seconde pour des élèves de nationalité française et étrangère, suivant la taille de la fratrie et le milieu d'origine

\begin{tabular}{|l|cccc|}
\hline CSP & 1 ou 2 enfants & 3ou 4 enfants & 5 enfants et plus & Ensemble \\
\hline Favorisée & 77 & 75 & 71 & $76 \%$ \\
Moyenne & 54 & 44 & 25 & $46 \%$ \\
Défavorisée & 39 & 29 & 18 & $29 \%$ \\
ensemble & 56 & 44 & 24 & $46 \%$ \\
\hline
\end{tabular}

Source : Ministère de l'Education, Direction de l'évaluation, Panel 1980.

Les conclusions qui découlent de l'examen du tableau ne sont pas liées à l'étape particulière du cursus retenu pour construire celui-ci. Le graphique 4, ci-contre, repris de $M$. Duthoit ${ }^{(30)}$ et issu de la même source, donne des conclusions analogues pour ce qui est de l'entrée en sixième. De façon nette, la réussite scolaire diminue avec l'augmentation de la taille de la fratrie, dès lors que celle-ci dépasse les deux enfants: être enfant unique ou n'avoir qu'un seul frère ou scur n'a pas d'effet notable sur la probabilité d'accéder à la classe supérieure ; avoir plus de frères et sœurs affaiblit les chances de continuer.

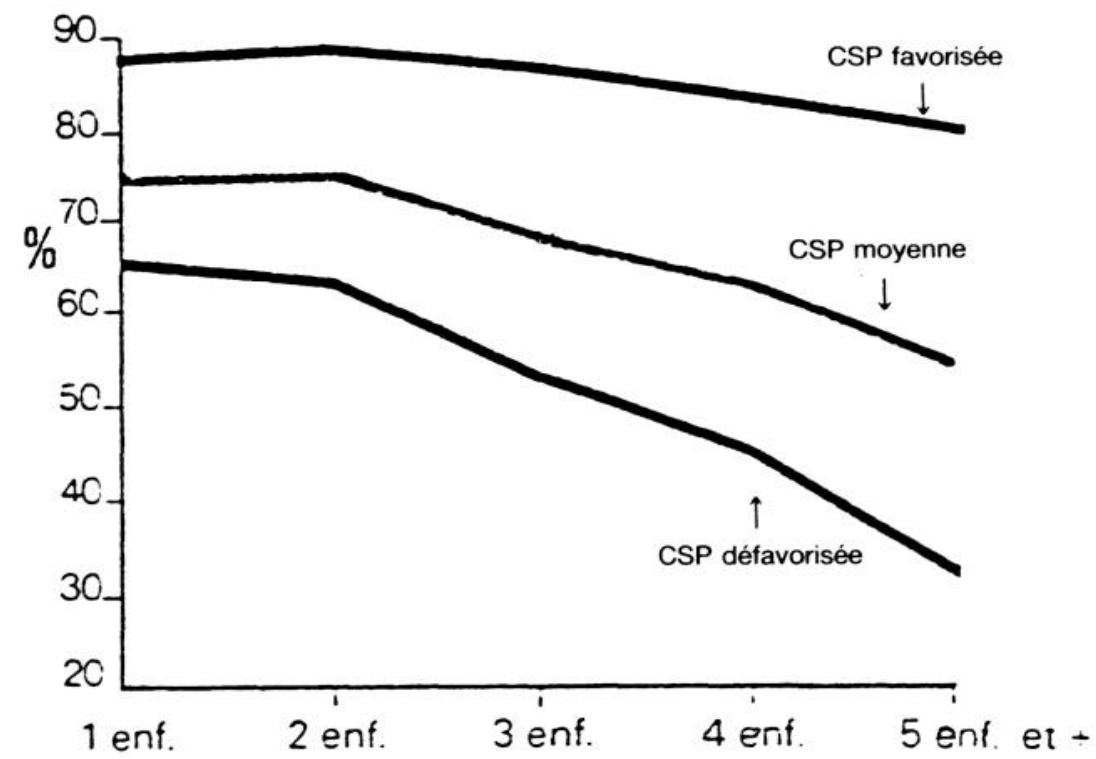

4. Taux d'accès en $6^{e}$ en 6 ans selon le nombre d'enfants dans la famille.

Source : M. Duthoit, " L'enfant et l'école " Education et Formation $n^{\circ} 16,1988$.

(30) M. Duthoit, 1988, “ L'enfant et l'école», Education et formation, $n^{\circ} 16$, juilletseptembre. 
Cet effet de la taille de fratrie dépend fortement du milieu social d'appartenance qui agit, en quelque sorte, comme un facteur protecteur: l'effet est faible pour les familles favorisées, important pour les familles défavorisées. Au total la présence d'un effet propre n'est pas niable. C'est bien le résultat qu'Edith Blake annonce.

\section{... et la baisse de la fécondité accompagnera vraisemblablement une augmentation du niveau scolaire moyen des Français}

Les chiffres utilisés décrivent les probabilités de passage dans une classe supérieure. De nombreux éléments interviennent pour expliquer cette probabilité, les capacités scolaires intrinsèques des enfants, leurs motivations, goûts et ambitions qui peuvent ou non leur suggérer, à capacité égale, de poursuivre leurs études; et la volonté familiale enfin de financer et d'obtenir une telle poursuite. II faut donc se garder d'interpréter trop mécaniqement en termes de causalité la liaison que l'on observe ${ }^{(31)}$.

Ceci dit, si on admettait que le modèle à deux enfants soit devenu la règle absolue et que donc, seules les différences de milieu social puissent expliquer les différences de réussite scolaire, le taux d'accès en seconde passerait de $46 \%$ à $53 \%$ et celui de la réussite au baccalauréat de $39 \%$ à $48 \%$. Pour obtenir ces chiffres, on a tout simplement calculé ce que serait le taux de réussite, à structure par catégorie sociale inchangée, si la réussite scolaire ne dépendait aucunement de la taille de la fratrie et s'alignait exactement sur ce que l'on observe pour les fratries les moins nombreuses. Ce calcul assez simple illustre bien cependant l'importance potentielle des phénomènes.

(31) La modélisation des comportements parentaux fait par exemple l'objet de nombreux travaux d'économistes visant précisément à expliquer la diminution de la fécondité par le développement du souci parental d'assurer une meilleure réussite à leurs enfants. (Cf. un exemple dans E.A. Hanushek "The Trade-off between Child Quantity and Quality ", Journal of Political Economy $n^{\circ} 1$, Vol. 100, Feb 1992). Meilleure réussite scolaire et baisse de la taille de la fratrie sont, dans le cas d'espèce, deux variables simultanément déterminées. 